\title{
$1.2 \mathrm{~mm}$ emission from Cen A
}

\author{
Zita Banhidi, Rolf Chini, Marcus Albrecht \\ Astronomisches Institut Ruhr-Universität Bochum, D-44780 Bochum, \\ Universitätsstraße 150, Germany
}

\begin{abstract}
Centaurus A is the closest active radio galaxy at a distance of around 3.5 Mpc. New $1.2 \mathrm{~mm}$ imaging performed with SIMBA at the Swedish ESO Submillimetre Telescope (SEST) in Chile gives an insight into the distribution of thermal and non-thermal emission. Apart from extended synchrotron emission in the radio lobes we have detected the unresolved AGN core and the characteristic dust lane.
\end{abstract}

\section{Observations}

The $1.2 \mathrm{~mm}$ continuum observations were obtained at the SEST using the 37channel bolometer array SIMBA with an angular resolution of $24^{\prime \prime} .28$ independent maps were observed between June 2001 and August 2002 in fast scanning mode. The data was reduced using the MOPSI software package developed by R. Zylka. The final image of Centaurus A has a residual noise of around $10 \mathrm{mJy}$ beam $^{-1}$.

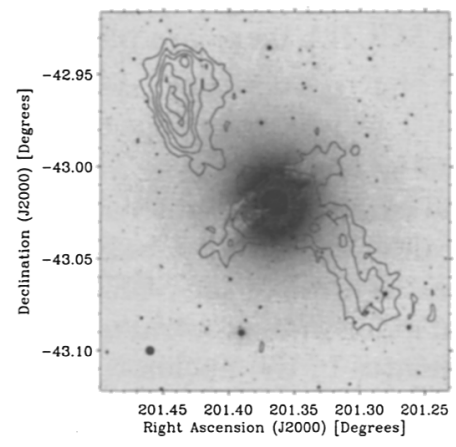

Figure 1. 2MASS J image overlayed with $1.2 \mathrm{~mm}$ contours (starting at $0.02 \mathrm{Jy}$ and increasing with steps of $0.04 \mathrm{Jy}$ ). (Atlas Image courtesy of 2MASS/UMass/IPAC-Caltech/NASA/NSF)

\section{Inner radio lobes}

By combining the $21 \mathrm{~cm}$ continuum emission image from Condon et al. (1996) with our $1.2 \mathrm{~mm}$ image we derive a map showing the spectral index across the lobes (see Fig. 2). The spectral index is found to vary between -0.8 and -1.5 . The corresponding radio spectral index $(20 \mathrm{~cm} / 6 \mathrm{~cm})$ taken from the literature 
ranges from -0.86 for the northern to -0.64 for the southern lobe with even higher values of -0.3 (for $18 \mathrm{~cm} / 6 \mathrm{~cm}$ ) in certain regions. This suggests that the emission from the lobes at $1.2 \mathrm{~mm}$ is entirely due to synchrotron radiation.
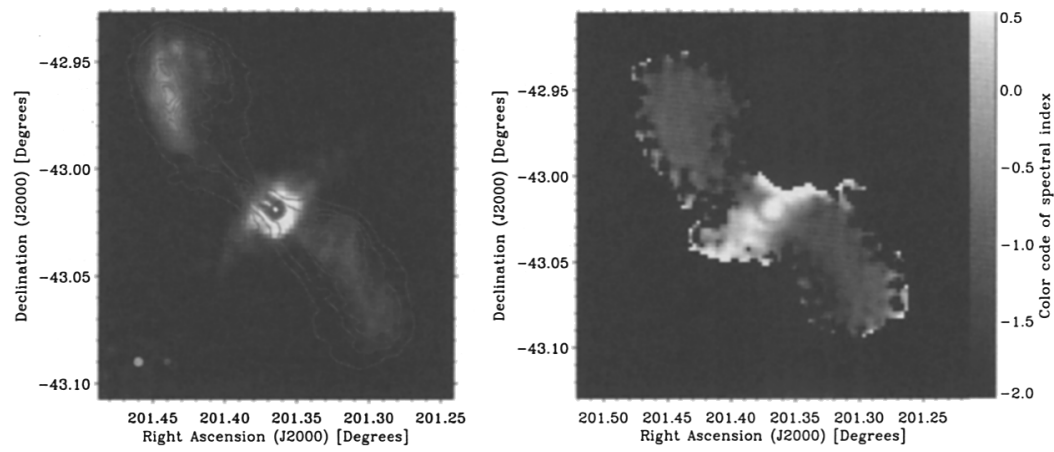

Figure 2. Left: $1.2 \mathrm{~mm}$ image overlayed with the $21 \mathrm{~cm}$ contours from Condon et al. (1996). Circles in the lower left corner represent the beam sizes (bright: $24^{\prime \prime}$ for $1.2 \mathrm{~mm}$ map, dark: $18^{\prime \prime}$ for $21 \mathrm{~cm}$ map). Contours start at a value of $0.1 \mathrm{Jy}$ and increase in steps of 0.5 Jy. Right: Spectral index $(21 \mathrm{~cm} / 1.2 \mathrm{~mm})$ across Centaurus A.

\section{Dust emission}

The thermal emission from the dust lane prominent in optical and infrared observations is also detected in the $1.2 \mathrm{~mm}$ map (see contours in Fig. 1). Comparisons with archival $450 / 850 \mu \mathrm{m}$ SCUBA maps are planned for the near future.

\section{Nucleus}

The $281.2 \mathrm{~mm}$ observations were spread over a time interval of more than 14 months. Photometry of the extremely bright nucleus of Centaurus A suggests flux variations on all timescales with amplitudes between $6.5 \mathrm{Jy}$ and $11.5 \mathrm{Jy}$ (the typical absolute flux calibration error of the SEST is estimated to $15 \%$ ). Nevertheless, the positive spectral index in the central region (see Fig. 2) demonstrates that thermal emission dominates in the nucleus.

\section{References}

Burns, J. O., Feigelson, E. D. \& Schreier, E. J. 1983, ApJ, 273, 128

Clarke, D. A., Burns, J. O. \& Norman, M. L. 1992, ApJ, 395, 444

Condon, J. J., Helou, G., Sanders, D. B. \& Soifer, B. T. 1996, ApJS, 103, 81

Nyman, L.-A., Lerner, M., Nielbock, M., et al. 2001, ESO Messenger, 106, 40 